\title{
TYPE-A CAROTID CAVERNOUS FISTULA: OPHTHALMOLOGICAL MANIFESTATIONS, MANAGEMENT AND OUTCOME OF TREATMENT IN 28 PATIENTS
}

\author{
Mary Thomas ${ }^{1}$, Deepika E2, Santhosh Joseph ${ }^{3}$ \\ ${ }^{1}$ Associate Professor, Department of Ophthalmology, SRMC \& RI, Porur, Chennai, Tamilnadu, India. \\ ${ }_{2}^{2}$ ost Graduate Student, Department of Ophthalmology, SRMC \& RI, Porur, Chennai, Tamilnadu, India. \\ ${ }^{3}$ Professor \& HOD, Department of Neuro-radiology, SRMC \& RI, Porur, Chennai, Tamilnadu, India.
}

ABSTRACT
BACKGROUND AND PURPOSE
Carotid cavernous fistula can present with myriad of symptoms and can often be misdiagnosed. The purpose of the study is to
evaluate different clinical manifestations, management and outcome of treatment in direct CCF (type-A) carotid cavernous fistula.

\section{MATERIALS AND METHODS}

A retrospective study of patients who were diagnosed and treated for Type-A CCF in a tertiary care centre attached to a Medical College in Chennai. The case records of 28 patients were analysed. History, clinical presentations and modality of treatment of each patient were recorded and clinical features were compared with that of the outcome.

\section{RESULTS}

Total of 28 patients from 9 years to 51 years were evaluated; 24 (85.7\%) patients had history of trauma and in 4 (14.3\%) patients CCF developed spontaneously; 25 (89.3\%) patients had undergone interventional procedure and 3 (10.7\%) had spontaneous thrombosis. None of the patients developed morbidity or mortality related to the procedure.

\section{CONCLUSION}

CCF should be included in the differential diagnosis of any atypical red eye, especially when associated with tinnitus. Ophthalmologist has an important role in the diagnosis of CCF and in the management of certain vision threatening complications like exposure keratopathy, secondary glaucoma or management of residual diplopia.

\section{KEYWORDS}

Carotid Cavernous Fistula, Direct Fistula, Dural Fistula, Micro-Catheter, Detachable Balloons.

HOW TO CITE THIS ARTICLE: Thomas M, Deepika E, Joseph S. Type-A carotid cavernous fistula: ophthalmological manifestations, management and outcome of treatment in 28 patients. J. Evolution Med. Dent. Sci. 2016;5(45):2836-2839, DOI: $10.14260 /$ jemds/2016/662

\section{INTRODUCTION}

Carotid Cavernous Sinus Fistula (CCF) is defined as an abnormal connection between the Cavernous Sinus (CS) and the carotid arterial system. The cavernous sinuses are located on either side of the sella turcica. Superior and inferior ophthalmic veins, (SOV, IOV) drain into CS which in turn drain into the superior and inferior petrosal sinuses [Figure 1]. The contents of cavernous sinus are the carotid artery; its sympathetic plexus; third, fourth and sixth cranial nerves; ophthalmic and maxillary division of the fifth nerve.

Barrow et al, have classified CCF as direct or indirect based on selective arteriography.(1) Type A or Direct fistula is a direct connection between the cavernous segment of Internal Carotid Artery (ICA) and the CS. Indirect shunts or dural fistula occur between CS and one or more branches of ICA (Type B), external carotid artery (Type C) or both (Type D).

Cavernous segment of the ICA is fixed to the dura mater, thus mobility is limited and is prone for shearing injury

Financial or Other, Competing Interest: None.

Submission 19-04-2016, Peer Review 15-05-2016,

Acceptance 21-05-2016, Published 06-06-2016.

Corresponding Author:

Dr. Mary Thomas,

Associate Professor

Department of Ophthalmology,

SRMC \& RI, Porur,

Chennai-600116,

Tamilnadu, India.

E-mail: marysanthoshj@yahoo.co.in

DOI: $10.14260 /$ jemds $/ 2016 / 662$ leading to stretch and tear of the ICA wall during traffic accidents. The onset of symptoms with a Type-A fistula is usually rapid due to high flow shunts and can be very dramatic, especially with aneurysmal rupture of spontaneous CCF. Of the direct CCFs, $70-90 \%$ are caused by tear or severe laceration in the cavernous segment of ICA following traffic accidents or fall.(2) They are more commonly seen in younger males.(3) In $1-2 \%$ of patients traumatic CCF leads to bilateral symptoms, because of inter-communicating CS drainage or even exists only on the side opposite the fistula due to variations in drainage patterns. ${ }^{(4-6)}$

$10-20 \%$ of direct fistula may be spontaneous due to rupture of aneurysm of cavernous part of ICA or weakening of the vessel wall as in Ehlers-Danlos syndrome or pseudoxanthoma elasticum.(7-9)

Due to high flow, pressure in the CS increases and venous flow alters in rate and direction in the draining/collecting veins and they become arterialised. Altered venous dynamics and subsequent reduction in arterial perfusion result in different clinical manifestations. The triad of symptoms in CCF include pulsatile proptosis, chemosis and bruit (Dandy's clinical triad), and patients with these features should clinch the possibility of CCF.(10)

Anteriorly draining fistula drain into orbital veins leading to arterialisation of veins and shows neuroophthalmological features. When blood flows posteriorly into superior and inferior petrosal sinus, it may manifest as isolated cranial nerve palsy. Intra-cerebral and subarachnoid haemorrhage related to venous hypertension in CCFs may be life-threatening.(11) 
Main indications for treatment of CCF include glaucoma, diplopia, intolerable bruit or headache and severe proptosis resulting in exposure keratopathy. Another indication is to prevent torrential and uncontrollable epistaxis, in case if there is a torn dura of the CS with herniation of the sinus into the nasopharynx as revealed in imaging.(12) Spontaneous closure from thrombosis of cavernous sinus is unlikely, especially in traumatic CCFs and in high flow fistula. Carotid compression therapy may also be successful in closure of slow flow fistulas $(17 \%$ of direct and $30 \%$ of dural fistula). In case if retrograde filling of cortical venous system is present compression is not indicated due to risk of intracranial haemorrhage.

Sacrificing the CA by carotid ligation was the treatment performed earlier. Interventional radiologic techniques with trans-arterially deployed detachable balloon embolization and maintenance of patent ICA is the gold standard in managing traumatic CCF (Close hole, keep artery open), but in some cases carotid sacrifice may be needed to occlude the fistula. Success rate of CCF occlusion by detachable balloon is found to be $90-98 \%$.(13) Stent assisted coil placement is done for high flow CCF that are associated with severe laceration of ICA with a combination of detachable balloons, coils or liquid embolic agents. If trans-arterial route is difficult, transvenous approach (Femoral route through the inferior petrosal sinus) or surgically through superior ophthalmic vein may be warranted. In this study, we present various clinical presentations and outcome of treatment in 28 patients diagnosed with direct CCF.

\section{MATERIAL AND METHODS}

A retrospective study of patients who were diagnosed and treated for Type-A CCF (Direct CCF) in a tertiary care centre attached to a Medical College in Chennai during a period of five years from May 2010 to June 2015. In our study, we had analysed the case records of 28 patients.

For all patients, complete evaluation including detailed history was taken. Best Corrected Visual Acuity (BCVA) was recorded with Snellen's chart and Ishihara's chart was used for colour vision checking. Slit lamp examination was done for all patients. Intraocular Pressure (IOP) was measured by Goldmann applanation tonometer and proptosis was measured with Hertel's exophthalmometer. Fundus examination was done using slit lamp biomicroscopy with a +78D lens and indirect ophthalmoscopy with +20D lens.

All patients were specially checked for chemosis, tortuosity and dilatation of conjunctival vessels, exposure keratopathy, anterior chamber depth, pupillary size, reaction including Relative Afferent Pupillary Defect (RAPD), glaucoma, vitreous haemorrhage, papilloedema/optic atrophy, status of the retinal vessels, extraocular muscle involvement, cranial nerve palsies. All findings were recoded and compared with those at outcome [Figure $1 \& 2$ ]. Patients were evaluated by the same ophthalmologist.

Diagnosis was confirmed by CT, Magnetic Resonance Angiography (MRA) or Carotid angiography and decision on treatment was made based on this radiological features. Digital Subtraction Angiography (DSA) road map (Philips Allura Xper FD 20-20 Biplane Neuro-angiography system, Amsterdam, Netherlands) was invaluable for microcatheter placement (Excelsior, Excel 14 or SL-10;
Boston Scientific, Natick, Massachusetts) and delivery of embolic materials. Different embolic materials used were Guglielmi detachable coils (GDC; Boston Scientific, Natick, Massachusetts), interlocking detachable coils (IDC; Boston Scientific, Natick, Massachusetts), Cook's detachable coils (CDC; Cook Inc.), Trufill DCS (Johnson \& Johnson) or detachable balloon [Figure 4]. Interventional procedures were done by the same Interventional radiologist.

Platinum coil was used for embolization in 13 (46.4\%), balloon in $9(32.1 \%)$ cases. Other cases were treated with balloon and coil or balloon with GDC and DCS. Balloon got deflated in $1(3.6 \%)$ and was managed by coils in the same sitting.

\section{RESULTS}

Total of 28 patients from 9 years to 51 years of age were treated. This included 22 males (78.6\%) and 6 females (21.4\%).

$24(85.7 \%)$ patients had history of trauma including a case with history of gunshot injury and another one with history of trauma with umbrella while playing, who developed gradual progression of proptosis and tinnitus over a period of 1 year. In all other patients, ophthalmological problems started in 2 weeks to 3 months after the trauma; 2 (7.14\%) post-traumatic patients had bilateral symptoms. In 4 patients (14.3\%), CCF developed spontaneously following aneurysmal rupture including one with Ehlers-Danlos syndrome.

Cranial nerve involvement $(3,4,5,6$ or 7$)$ was present in 9 patients (32.14\%). Extraocular movement restriction was not only due to nerve involvement, but mechanical restriction also contributed as evidenced by the thickening of the muscles seen in MRI. Double vision was present in 7 (25\%), bruit was present in $9(32.14 \%)$, secondary glaucoma in 4 (7.1\%), papilloedema in $2(7.1 \%)$, primary optic atrophy in 7 (25\%) with Relative Afferent Pupillary Defect (RAPD) and visual acuity ranging from $3 / 60$ to no perception of light [Table 1].

$19(67.9 \%)$ patients cured by single attempt of intervention [Figures 5 \& 6]; 6 (21.4\%) needed second attempt and $3(10.7 \%)$ cured by spontaneous thrombosis.

Tinnitus and bruit disappeared immediately after the procedure, proptosis and chemosis took a few days' time and nerve palsies took a few months for complete recovery. None of them had to undergo extraocular muscle surgery.

None of the patients developed morbidity or mortality related to the procedure. All patients regained 6/9 to $6 / 6$ vision except those with pre-existing optic atrophy, corneal opacity and severe damage of the eye as in the gunshot injury case.

One patient showed pseudo-Argyll Robertson pupil at follow-up with a dilated pupil, which contracts only on adduction due to the misdirection of fibers to the pupil from the regenerating sprouts of $3 \mathrm{rd}$ nerve from the axons that previously innervated medial rectus.

Another patient with exposure keratopathy and Hand Movements (HM) vision at presentation improved to $6 / 36$ after the procedure and underwent keratoplasty later.

The boy with gunshot injury had uveitis and optic atrophy developed enophthalmos later. 


\begin{tabular}{|c|c|c|c|}
\hline Clinical Features & & No. & $\begin{array}{c}\text { \% of } \\
28\end{array}$ \\
\hline \multirow[b]{2}{*}{ Side of signs } & Unilateral & 26 & 92.9 \\
\hline & Bilateral & 2 & 7.1 \\
\hline Defective vision & & 14 & 50 \\
\hline Double vision & & 7 & 25 \\
\hline \multirow{4}{*}{ Orbital signs } & Proptosis & 27 & 96.4 \\
\hline & Conjunctival chemosis & 14 & 50 \\
\hline & $\begin{array}{l}\text { Cork screwing of- } \\
\text { conjunctival vessels }\end{array}$ & 28 & 100 \\
\hline & $\begin{array}{l}\text { Extraocular muscle- } \\
\text { involvement }\end{array}$ & 21 & 75 \\
\hline \multirow{5}{*}{$\begin{array}{l}\text { Cranial nerve } \\
\text { involvement }\end{array}$} & Optic atrophy & 7 & 25 \\
\hline & Third nerve & 4 & 14.3 \\
\hline & Fifth nerve & 1 & 3.6 \\
\hline & Sixth nerve & 6 & 21.4 \\
\hline & Facial nerve & 1 & 3.6 \\
\hline $\begin{array}{c}\text { Exposure } \\
\text { keratopathy }\end{array}$ & & 3 & 10.7 \\
\hline Pupillary & Dilated pupil & 6 & 21.4 \\
\hline involvement & RAPD & 7 & 25 \\
\hline Bruit & & 9 & 32.14 \\
\hline \multirow{5}{*}{ Fundus changes } & Disc oedema & 2 & 7.1 \\
\hline & Optic atrophy & 7 & 25 \\
\hline & Venous dilatation & 21 & 75 \\
\hline & Retinal oedema & 2 & 7.1 \\
\hline & Retinal haemorrhages & 2 & 7.1 \\
\hline Sec. glaucoma & & 4 & 14.3 \\
\hline & ents with Direct CCF & & \\
\hline
\end{tabular}

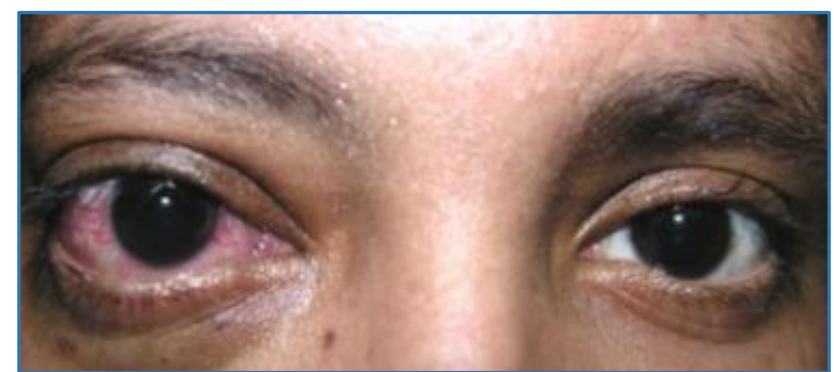

Fig. 3: Post-Procedure Photograph - 2nd Day

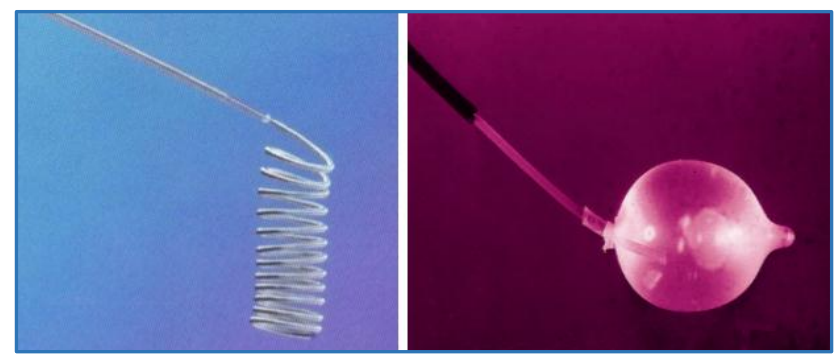

Fig. 4: Detachable Coil and Balloon

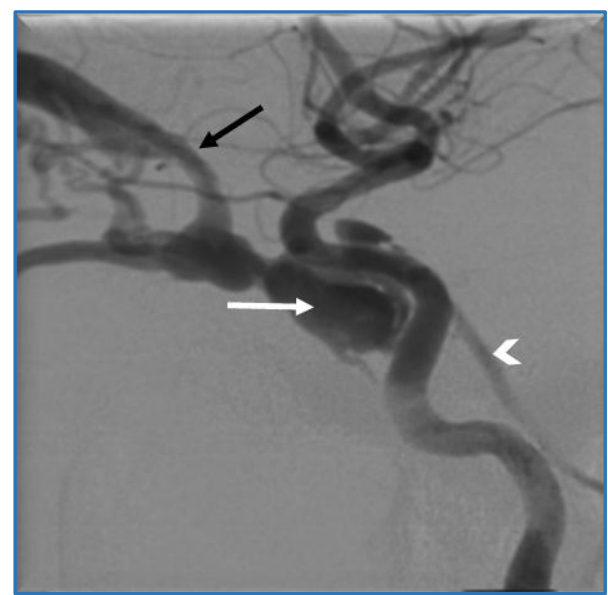

Fig. 5: Internal Carotid Angiogram Lateral View showing: (1) Type-A CCF with Filling of Cavernous Sinus from ICA (White Arrow); (2) Anterior Drainage to Superior Ophthalmic Vein (Black Arrow); (3) Posterior Drainage to Inferior Petrosal Sinus (White Arrow Head)

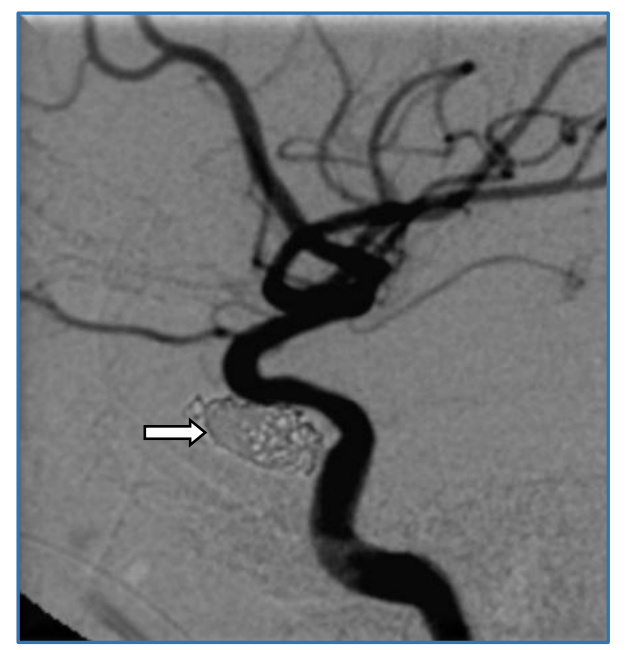

Fig. 6: Post-Procedure Angiogram showing Coil in the Cavernous Sinus (White Arrow) Without Any Fistula Proptosis and Chemosis

Fig. 2: Pre-Procedure Clinical Photograph showing

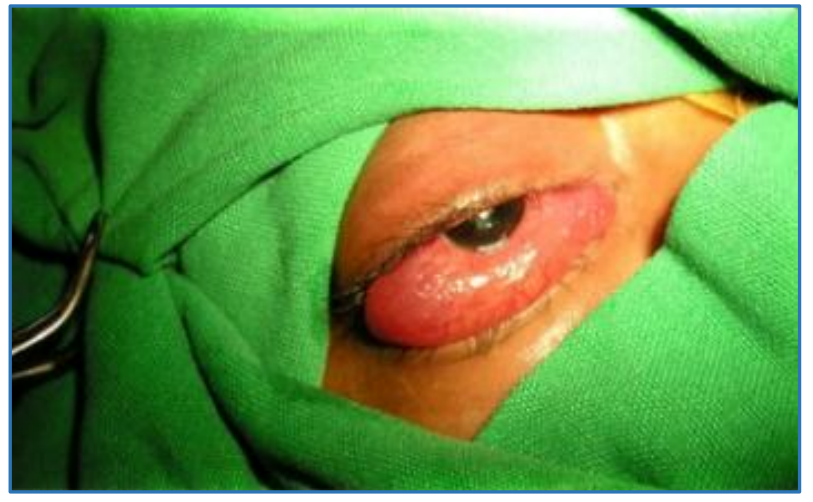




\section{DISCUSSION}

As Kurata emphasised, Dandy's classic triad of CCF (Proptosis, bruit and conjunctival chemosis) were not universally present in our patients also.(14) In our study proptosis was present in 27 (96.4\%), bruit in 9 (32.1\%) and conjunctival chemosis in 14 (50\%). Cork screwing of conjunctival vessels was present in all (100\%) cases.

$80 \%$ of cases, CCFs are very easily missed initially. With increased flow rate within the fistula and features of orbital congestion in cases of an anteriorly draining fistula may be wrongly diagnosed as painful ophthalmoplegia (Kosmorsky 1988), venous stasis retinopathies, Central Retinal Vein Occlusion (CRVO) or exudative retinal detachment due to wrong interpretation of the clinical signs or with features of isolated cranial nerve palsy (Miyachi 1993) in cases of a posteriorly draining fistula. This is more common with dural fistulas (Type B, C, D), because in dural fistulas the manifestations are much subtler with a slow and spontaneous onset. Two of our patients were treated elsewhere as allergic conjunctivitis and one patient attributed the chemosis to pesticide falling into the eye.

Patients with CCF may initially present to an ophthalmologist for their eye symptoms.(15) Careful history, eye examination and imaging modalities lead to correct and timely diagnosis and help to differentiate it from arteriovenous malformations, cavernous sinus thrombosis, orbital and cavernous sinus tumours, tumours of the base of the skull, mucocoele, thyroid ophthalmopathy.(16,17) Retrobulbar tumours, haemorrhages (Especially after trauma), infections like orbital cellulitis. Mucormycosis also can mimic CCF. Thyroid eye disease, orbital pseudotumours, Tolosa-Hunt syndrome can also be included in the differential diagnosis of CCF.(17)

6th nerve was the most commonly affected cranial nerve in our study - seen in $6(21.4 \%)$ patients, may be because of its free floating location within the CS.(18) Ophthalmic division of 5 th nerve and 7 th nerve palsy was seen in $1(3.6 \%)$ each; 7 th nerve involvement may be due to increased venous pressure or rarely due to arterial steal phenomenon.(19)

\section{CONCLUSION}

Carotid cavernous fistula, which may present with a myriad of nonspecific manifestations. CCF should be included in the differential diagnosis of any atypical red eye. Subjective complaint of hearing abnormal sounds within the head is an important clue. Identification of cork screw conjunctival and episcleral vessels, eliciting bruit on auscultation should raise the possibility of CCF and prompt diagnostic imaging should be undertaken.

On CT scan of the brain features of CCF like extraocular muscle thickness, dilated superior ophthalmic vein and prominent cavernous sinus should be looked for. Endovascular technique of treatment can give high cure rate and minimum complications.

Ophthalmologist should manage exposure keratopathy, should identify different causes of glaucoma like raised episcleral venous pressure, angle closure or iris neovascularization and should control IOP. Also should follow up these patients after interventional procedures for persistence of any of these problems to prevent vision loss. Persistent diplopia after resolution of CCF may need occlusion, appropriate prism or surgery.

\section{REFERENCES}

1. Barrow DL, Spector RH, Braun IF, et al. Classification and treatment of spontaneous carotid cavernous fistula. J Neurosurg 1985;62(2):248-56.
2. Yanoff M, Duker JS. Ophthalmology. Mosby: Spain, 2004; $2^{\text {nd }}$ ed:1403-4.

3. Locke CE. Intracranial arteriovenous aneurysm or pulsating exophthalmos. Ann Surg 1924;80(2):272-85.

4. Higashida RT, Halbach VV, Tasi FY, et al. Interventional neurovascular treatment of carotid and vertebral lesion: results in 324 cases. Am J Roentgenol 1989;153(3):57782.

5. Lewis AI, Tomsick TA, Tew JM. Management of 100 consecutive direct carotid-cavernous fistulas: results of treatment with detachable balloons. Neurosurgery 1995;36(2):239-44.

6. Santhosh J, Sanjay S. Radiology in neuro-ophthalmology. Endovascular interventions in ophthalmology. Neuroophthalmology aravind eye hospital 1995;3rd ed:275.

7. Taki W, Nakahara I, Nishi S, et al. Pathogenetic and therapeutic considerations of carotid- cavernous sinus fistula. Acta neurochir (Wien) 1994;127(1-2):6-14.

8. Bradac GB, Bender A, Curio G, et al. Report of two spontaneous direct carotid-cavernous fistula. Diagnostic and therapeutic considerations. Neuroradiology 1985;27(5):436-9.

9. Kashiwaga S, Tsuchida E, Goto K, et al. Balloon occlusion of a spontaneous carotid-cavernous fistula in ehlersdanlos syndrome type 4. Surg Neurol 1993;39(3):18790 .

10. Guptha S, Thakur AS, Bhardwaj N, et al. Carotid cavernous sinus fistula presenting with pulsating exophthalmos and secondary glaucoma. J Indian Med Assoc 2008;106(5):312-46.

11. Van Der Schaaf IC, Brilstra EH, Buskens E, et al. Endovascular treatment of aneurysms in the cavernous sinus: a systematic review on balloon occlusion of the parent vessel and embolization with coils. Stroke 2002;33(1):313-8.

12. Debrun GM, Lacour P, Fox JA, et al. Traumatic carotid cavernous fistulas: aetiology, clinical presentation, diagnosis, treatment, results. Semin Interventional Radiol 1987;4(4):242-8.

13. Santhosh J, Rao VR, Ravimandalam K, et al. Endovascular management of carotid cavernous fistulae: observation on angiographic and clinical results. Acta Neurol Scand 1993;88(5):320-6.

14. Kurata A, Takano M, Tokiwa K, et al. Spontaneous carotid- cavernous fistula presenting only with cranial nerve palsies. Am J Neuroradiol 1993;14(5):1097-101.

15. Miller NR. Diagnosis and management of dural carotidcavernous sinus fistulas. Neurosurg Focus 2007;23(5):E13.

16. Ohtsuka K, Hashimoto M. Clinical findings in a patient with spontaneous arteriovenous fistulas of the orbit. Am J Ophthalmol 1999;127(6):736-7.

17. Oestreicher JH, Frueh BR. Carotid-cavernous fistula mimicking graves' eye disease. Opthal Plast Reconstr Surg 1995;11(4):238-44.

18. Kanski JJ Orbit. Clinical ophthalmology a systematic approach. Butterworth heinemann Elsevier, editors. Philadelphia, USA 2006;p 184.

19. Moster ML, Sergot RC, Grossman RI. Dural carotidcavernous sinus vascular malformation with facial nerve paresis. Can J Ophthalmol 1988;23(1):27-9. 